\title{
Carbohydrate and protein recovery mixtures with or without added galactose does not improve subsequent high-intensity cycling performance
}

\author{
E. Warrick, R. F. G. King, J. P. O'Hara, N. Reeve and H. Luke \\ Leeds Metropolitan University, Leeds LS6 3QS, UK
}

Carbohydrates $(\mathrm{CHO})$ effectively restore glycogen post-exercise ${ }^{(1)}$. Some studies have also suggested that adding protein (PRO) to a CHO drink may further improve the recovery rate. This improvement may be a result of the regulatory effect of insulin on glucose uptake, particularly pronounced in the first hours of recovery ${ }^{(2)}$. There is currently no consensus between studies about how this effect may benefit subsequent exercise performance. As hepatic glucose output can increase up to seven- to tenfold with more vigorous activity ${ }^{(3)}$, maximising restoration of liver glycogen during recovery may further improve post-recovery performance. Galactose (GAL) is believed to preferentially restore liver rather than muscle glycogen ${ }^{(4)}$. The aim of the study was to investigate the effect of adding GAL to a CHOPRO mixture on post-recovery cycling performance.

Five well-conditioned men (age 33.4 (SE 6.9) years; body mass (BM) 77.7 (sE 5.3) kg; $\mathrm{V}_{\mathrm{O} 2 \max } 54.2$ (sE 6.3) $\mathrm{ml} / \mathrm{kg} \mathrm{BM}$ per min) completed a depletion protocol on three occasions after an overnight fast. The exercise was followed by a $4 \mathrm{~h}$ recovery period during which every $30 \mathrm{~min}$ a maltodextrin (MD)-GAL-PRO $(0.5 \mathrm{~g} \mathrm{MD}+0.3 \mathrm{~g}$ GAL $+0.2 \mathrm{~g}$ whey protein hydrolysate $+0.1 \mathrm{~g}$ L-leucine $+0.1 \mathrm{~g}$ phenylalanine/kg BM per h), MD-PRO $(0.8 \mathrm{~g} \mathrm{MD}+0.4 \mathrm{~g}$ PRO $/ \mathrm{kg} \mathrm{BM}$ per h) or MD (1.2 g/kg BM per h) drink was administered, randomly assigned under double-blind conditions. Subsequently, a cycle test to volitional fatigue at $85 \% \mathrm{~V}_{\mathrm{O} 2 \mathrm{max}}$ was performed. Venous blood samples were drawn at rest and every $30 \mathrm{~min}$ of recovery and analysed for plasma glucose and serum insulin concentrations. All data are expressed as means with their standard errors. Repeated measures ANOVA was used (significance $P \leq 0.05$ ).

The mean time to exhaustion after ingestion of MD-PRO (14 min $57 \mathrm{~s}$ ( $\mathrm{sE} 7 \mathrm{~min} 54 \mathrm{~s}$ )) was higher compared with MD (12 min $47 \mathrm{~s}$ (SE $7 \mathrm{~min} 54 \mathrm{~s})$ ) and MD-GAL-PRO (13 $\min 56 \mathrm{~s}(\mathrm{SE} 6 \mathrm{~min} 42 \mathrm{~s})$ ) condition, 17 and $7 \%$ respectively, but this difference was not significant. Plasma glucose and serum insulin concentrations during the recovery are shown in Figs. 1 and 2 . The only significant difference detected was in serum insulin concentrations at 120 min of recovery between MD and MD-PRO conditions $(P \leq 0.05)$.

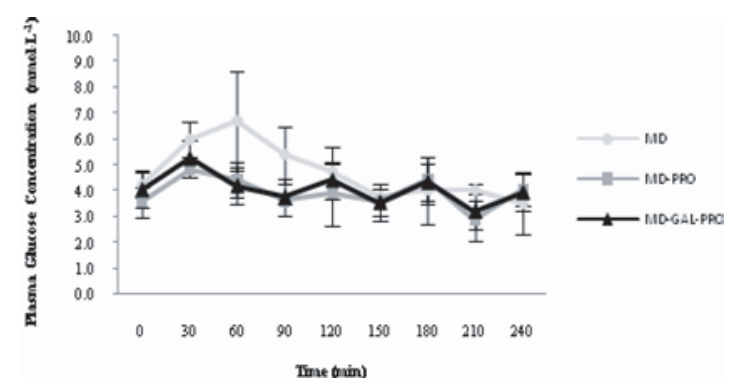

Fig. 1. Plasma glucose concentrations during the recovery. Values are means with their standard errors represented by vertical bars.

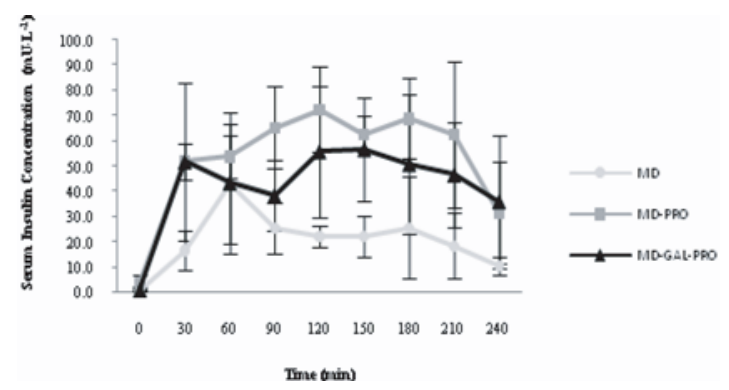

Fig. 2. Serum insulin concentrations during the recovery. Values are means with their standard errors represented by vertical bars.

These results suggest that adding GAL to a CHO-PRO recovery mixture does not improve post-recovery time to exhaustion. As expected, GAL attenuated the insulin response but even with high serum insulin concentrations after ingestion of MD-PRO the performance still was not enhanced. Further testing and direct measurement of both liver and muscle glycogen is required to clarify the effects of GAL on high-intensity cycling post-recovery.

1. Coyle EF (1991) J Sports Sci 9, 29-52.

2. Van Loon LJ, Saris WHM, Kruijshoop M et al. (2000) Am J Clin Nutr 72, 106-111.

3. Cooper DM, Barstow TJ, Bergner A et al. (1989) Am J Physiol 257, E405-E412.

4. Cohn RM \& Segal S (1973) Metab Clin Exp 22, 627-642. 